\title{
Conjunction of anti-parallel and component reconnection at the dayside MP: Cluster and Double Star coordinated observation on 6 April 2004
}

\author{
J. Wang, ${ }^{1,2}$ Z. Y. Pu, ${ }^{1}$ S. Y. Fu, ${ }^{1}$ X. G. Wang, ${ }^{3}$ C. J. Xiao, ${ }^{3}$ M. W. Dunlop, ${ }^{4}$ Y. Wei, ${ }^{1}$ \\ Y. V. Bogdanova, ${ }^{5}$ Q. G. Zong, ${ }^{1}$ and L. Xie ${ }^{1}$ \\ Received 17 February 2011; revised 24 March 2011; accepted 29 March 2011; published 21 May 2011.
}

[1] Previous theoretical and simulation studies have suggested that the anti-parallel and component reconnection can occur simultaneously on the dayside magnetopause. Certain observations have also been reported to support global conjunct pattern of magnetic reconnection. Here, we show direct evidence for the conjunction of anti-parallel and component MR using coordinated observations of Double Star TC-1 and Cluster under the same IMF condition on 6 April, 2004. The global MR X-line configuration constructed is in good agreement with the "S-shape" model. Citation: Wang, J., Z. Y. Pu, S. Y. Fu, X. G. Wang, C. J. Xiao, M. W. Dunlop, Y. Wei, Y. V. Bogdanova, Q. G. Zong, and L. Xie (2011), Conjunction of anti-parallel and component reconnection at the dayside MP: Cluster and Double Star coordinated observation on 6 April 2004, Geophys. Res. Lett., 38, L10105, doi:10.1029/2011GL047125.

\section{Introduction}

[2] Introduced first by Dungey [1961], magnetic reconnection (MR), which opens the magnetopause (MP) and provides a channel of mass and energy exchange between the magnetosphere (MSP) and the magnetosheath (MSH), has become one of the most fundamental basis of space physical studies [see, e.g., Paschmann, 2008].

[3] Taking into account the MSP asymmetry and nonzero IMF $B_{Y}$ and $B_{X}$ components that modifies purely southward/northward IMF conditions, two different scenarios were raised to explain the observed patterns of dayside MP reconnections. Based on the reconnection model with no guide field, the "anti-parallel reconnection" model [Crooker, 1979] suggested that the dayside MR occurred at the locations where the MSP and MSH magnetic fields were anti-parallel, forming two separate reconnection $\mathrm{X}$-lines in different hemispheres. However, the "component reconnection" model [Cowley, 1973] predicted that MR occurred near the subsolar point where the solar wind first encountered the MSP, extending continuously along the guild field direction to form a tilted reconnection X-line on the MP. Both sce-

\footnotetext{
${ }^{1}$ School of Earth and Space Sciences, Peking University, Beijing, China.

${ }^{2}$ Institute of Telecommunication Satellite, CAST, Beijing, China.

${ }^{3}$ School of Physics, Peking University, Beijing, China.

${ }^{4}$ Space Science and Technology Department, Rutherford Appleton Laboratory, Chilton, UK.

${ }^{5}$ Mullard Space Science Laboratory, University College London, Dorking, UK.
}

Copyright 2011 by the American Geophysical Union. 0094-8276/11/2011GL047125 narios were proved by observations [see, e.g., Gosling et al., 1990; Chandler et al., 1999; Fuselier et al., 2000; Onsager et al., 2001; Pu et al., 2005].

[4] Component and antiparallel MR scenarios were later found to be related and tend to co-exist in the theoretical studies of Moore et al. [2002] and Dorelli et al. [2007]. In observation studies, using 3D plasma data from Polar observations, Trattner et al. [2007] concluded that the two models could both work at the MP, depending on the specific IMF conditions, and $P u$ et al. [2007] carried out a statistical study of 290 accelerated flows in both low and high latitudes under predominantly dawnward IMF conditions. Examples of both anti-parallel and component MR were revealed in this study under similar IMF clock angles. By characterizing magnetic null points, Dunlop et al. [2009] reported a highlatitude anti-parallel MR event accompanied with a lowlatitude FTE. Nevertheless, direct case by case evidences are requisite for this issue.

[5] In this paper, we show a direct evidence for co-existence of anti-parallel and component MR with a detailed investigation on 6 April 2004 event when the IMF remained stable for over 2 hours [Dunlop et al., 2005; Wang et al., 2008]. During the event, FTEs observed by TC-1 were very likely generated via component MR and less than an hour later, Cluster passed by a MR region where observations favored the anti-parallel configuration. Features of a global MR $\mathrm{X}$-line on the MP were also obtained.

\section{Observations}

[6] As shown in Figure 1, both TC-1 and Cluster were located near the dayside MP. Figure 2 gives the IMF condition and magnetic field, plasma observations. As presented by 1-min spacecraft-interspersed data set at the bow shock nose (the High Resolution OMNI data set, See ftp:// nssdcftp.gsfc.nasa.gov/spacecraft_data/omni/high_res_omni and related documents), the IMF remained southward with strong duskward $B_{Y}$ from $\sim 03: 30$ UT for more than 2 hours and the solar wind bulk velocity remained above $500 \mathrm{~km} / \mathrm{s}$. As indicated by vertical black lines, TC- 1 crossed the MP at 04:13 UT, outbound from MSP to MSH, while Cluster tetrahedron was also traveling outward and encountered the MP at 04:34 UT, and all spacecrafts (SCs) stayed in the MSH until the end of this period.

[7] It is clear that $B_{N}$ bipolar signatures were observed in the adjacent MSH. Together with the enhanced $|B|$, the reduced ion density and increased temperature, it is easy to characterize that both TC-1 and Cluster encountered several FTEs in the interval, as indicated by dashed lines. The HIA 

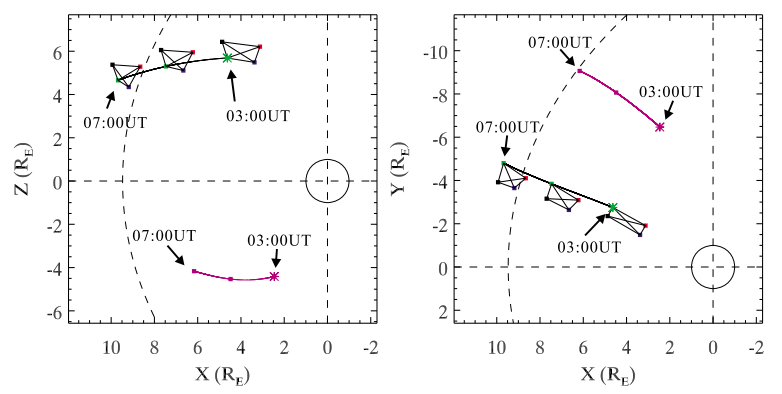

Figure 1. The locations and orbits of Double Star TC-1 and Cluster. The Cluster orbit also shows the SC configurations (scaled up by a factor of 30). The black dashed line indicates the MP, based on Shue model.

energy spectrograms from TC-1 and Cluster $\mathrm{C} 3$ also showed possible mixing of MSP and MSH plasmas, in agreements with the FTE crossings. As stated in previous work [Dunlop et al., 2005], these FTEs were very likely generated around the subsolar region via component reconnection.

[8] It is noteworthy to see that the last $B_{N}$ bipolar observed by Cluster at 05:20 UT, indicated by blue solid line in Figure 2, was quite different from the others. After removing an averaged flow speed, a flow reversal over $\pm 150 \mathrm{~km} / \mathrm{s}$ in the $L$ direction is revealed, in corresponding to $B_{N}$ bipolar signature, as indicated by vertical black lines in Figure 3a. Right at the moment of the jet peaks, ion heating was observed. It is very likely that the SC encountered an MR site.

[9] Although the encounter of MP was nearly an hour ago, as indicated in Figure 2, the plasma velocity perpendicular to the MP remained around zero for the whole period, thus we can reasonably conclude that the SC was departing from the MP very slowly and the distance between the SC and the MP was quite limited. Which is more important, the OMNI data show a continuous IMF with no evidence of magnetic shear which may induce reconnection within the MSH magnetic fields. This implies that the only possible site of the magnetic shear, i.e., the source of the $\mathrm{MR}$, is the MP.

\section{Detailed Analysis}

\subsection{FTEs and Low Latitude Component MR}

[10] The maximum and minimum variance analysis [Sonnerup and Scheible, 1998] and the deHoffmann-Teller analysis [Khrabrov and Sonnerup, 1998] were applied to obtain orientations and motions of the two TC-1 FTEs. The results are listed in Table 1, consistent with Dunlop et al. [2005]. The axis "clock angle" is defined clockwise from GSM $+Z$ direction to the FTE axis [Wang et al., 2008]. The "core field" is given as the maximum of magnetic field along the FTE axis.

[11] In the previous work of Dunlop et al. [2005], the TC-1 FTEs were very likely to be generated around the subsolar region by a tilted X-line. Taking that the SC was located $\sim 9 R_{E}$ away from the subsolar point and the velocities of FTEs $(122 \sim 171 \mathrm{~km} / \mathrm{s}$ in the $X-Y$ plane), we could then roughly obtain that the FTEs were produced $5 \sim 8 \mathrm{~min}$ before being observed, i.e., at 04:20 04:30 UT, when the IMF clock angle was $\sim-140^{\circ}$. The MR location and the IMF condition, together with the observation of strong FTE "core field" $(50 \sim 70 \mathrm{nT})$, lead to the conclusion that the MR was a component one.

\subsection{Fast Flows and High Latitude Anti-parallel MR}

[12] As presented in Figure 3b, the Walén test was also applied to the period of 05:20 05:21 UT and yielded reasonably good Walén relations, indicating a pair of oppositely propagating Alfvén waves observed by $\mathrm{C} 1$ and C3 simultaneously, and implying an MR occurring nearby.

[13] All the above results confirmed that an MR event occurred near the location of Cluster C3. Although a very clear mixture of MSP and MSH plasma was observed, it is worthy to mention that neither clear Hall effect nor "D-shape" distribution was found, possibly due to the fact that $\mathrm{C} 3$ was passing over the reconnection area in the MSH at a distance away from the magnetopause current sheet, rather than a traversal through the major MR region.

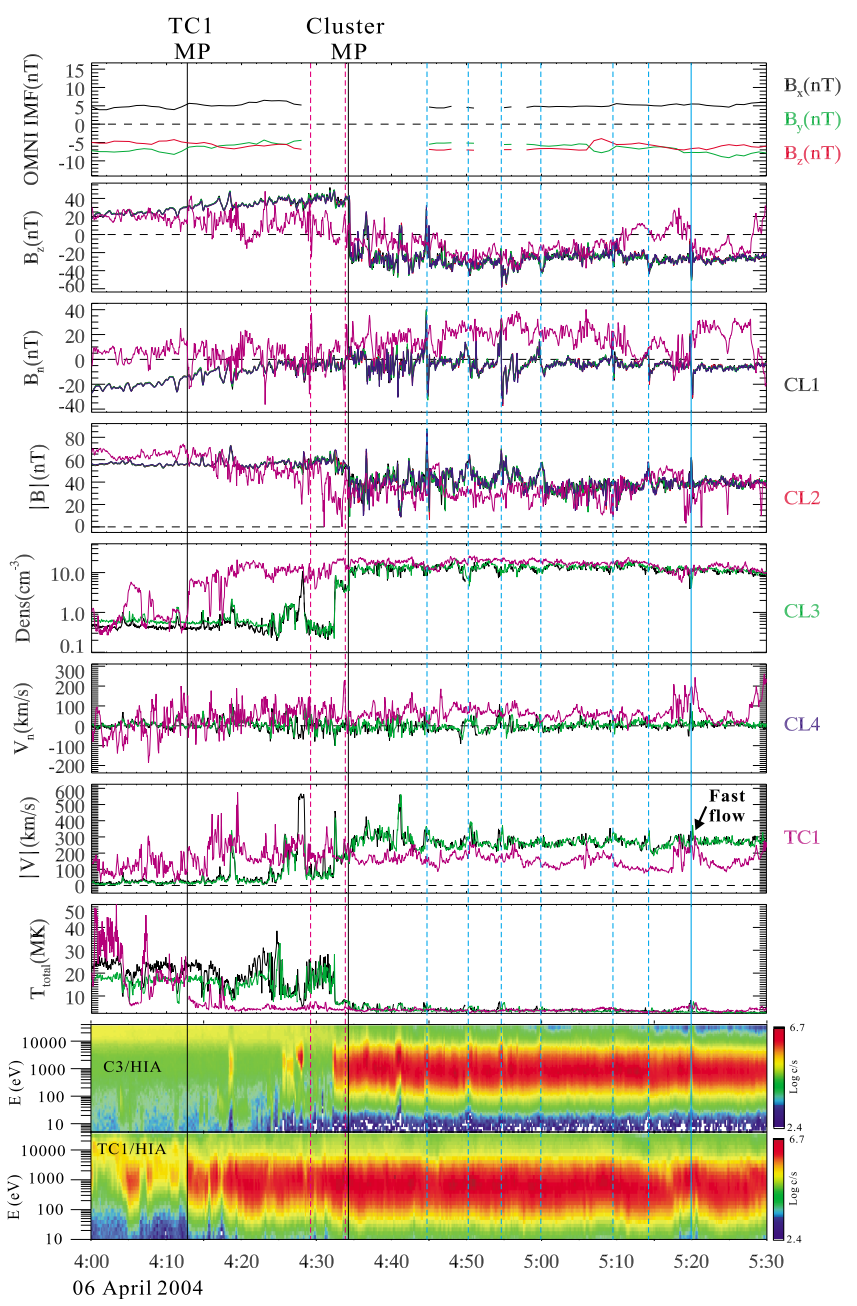

Figure 2. The observations of cluster and TC-1 from 04:00 UT to 05:30 UT, 6 April 2004. From top to bottom, OMNI IMF data, $B_{Z}$ in GSM, $B_{N}$ in boundary normal coordinates (BNC), total magnetic field, hot ion density, $V_{N}$ in $\mathrm{BNC}$, total velocity, temperature, and energy spectra of C3/HIA and TC-1/HIA. The vertical black line indicates the MP crossing of TC- 1 and Cluster and the magenta blue dashed line shows the $B_{N}$ bipolar observed, respectively, by TC-1 and Cluster. The fast flow observed by Cluster at 05:20 UT is also pointed out. 


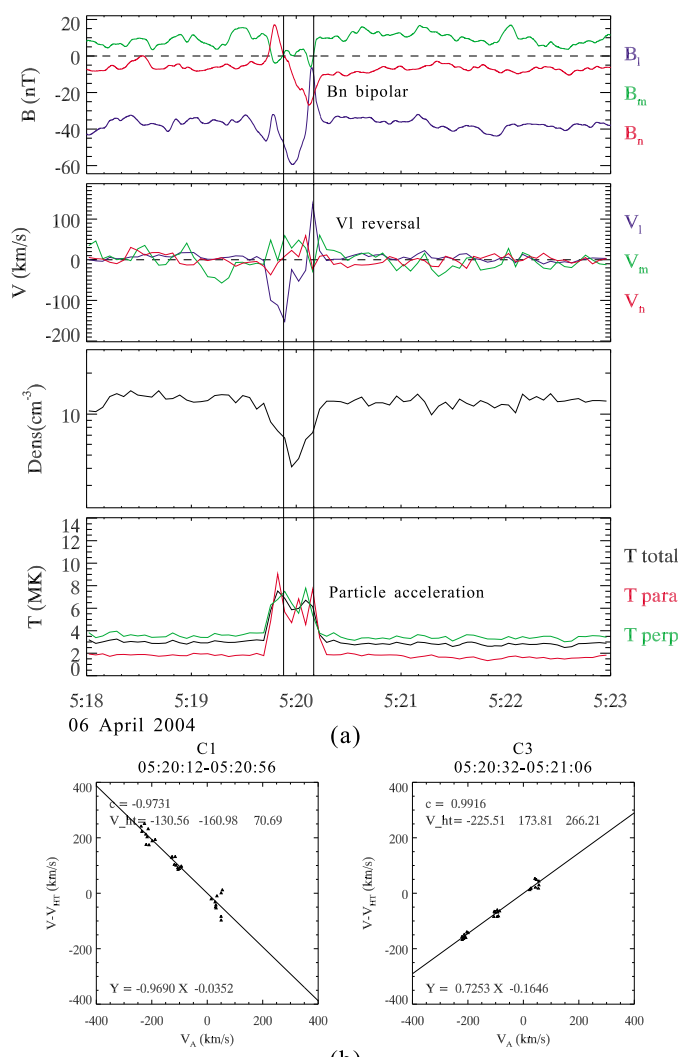

(b)

Figure 3. The observations of Cluster/C 3 on the $\mathrm{MR}$ region on 6 April 2004. (a) From top to bottom, magnetic field, velocity, density and temperature. (b) Walén results during 05:20 05:21 UT from C1 and C3.

[14] Besides, the peak velocity of outflow jet can be estimated as $(47.0,-74.1,-67.5)$ and $(-83.0,76.6,93.6) \mathrm{km} / \mathrm{s}$ (GSM) and at 05:20 UT and the local MSH field was (10.3, $-24.3,-28.8) \mathrm{nT}$ (GSM). Geometry studies show that the angles between the MSH field and the two jets are $12.1^{\circ}$ and $160.8^{\circ}$, indicating that these two fast jets were, respectively, almost parallel and anti-parallel to the IMF.

[15] In the component MR scenario, a notable guide field is present in the MR X-line direction, so the magnetic field is not perpendicular to the $\mathrm{X}$-line and thus obviously not parallel to the outflow jets. Based on the observations and calculations above, it is reasonable to infer that the MR event, which Cluster passed by and observed distantly, was essentially anti-parallel.

\subsection{Global Pattern of Reconnection}

[16] Although the observations on FTEs and the flow reversal were about an hour apart, the IMF varied little during this period of time, with only less than $10^{\circ}$ of the IMF clock angle variation. These two MR events can be considered happening under the same IMF condition. This

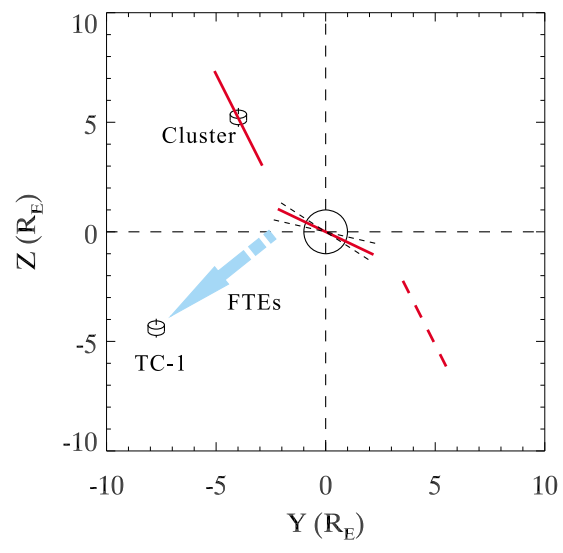

Figure 4. The obtained configuration of dayside MR Xline, in good agreement with predicted "S-shape" model. The red solid lines gives the $\mathrm{X}$-lines in low latitude and northern high latitude, and the red dashed line the mirror in southern high latitude. The two dashed black lines present the FTE axes.

result agrees very well with the simulation of Berchem et al. [2008].

[17] We have also constructed the global configuration of the dayside MR X-line under this IMF orientation, as shown in Figure 4. Based on the FTE generation theories [Lee and Fu, 1985; Scholer, 1988; Wang et al., 2008], the FTE axis should be along the X-line at the location of generation. Following Dunlop et al. [2005], the FTEs observed by TC-1 are not far away from the generation site, thus we average the "clock angle" of the two FTE axes and regarded it as the $\mathrm{X}$-line orientation at low latitudes (with standard deviation of $\sim 17^{\circ}$ ). At the northern high latitudes, the $X$-line should elongate along the MP and perpendicular to the reconnection plane. Therefore, by solving the over-determined equation set $\mathbf{n} \cdot \mathbf{A}=0$ (A presenting the local MSH magnetic field, the two jets and the MP normal direction, respectively), we obtain the most perpendicular direction $\mathbf{n}$ as the high latitude $\mathrm{X}$-line orientation. With the absence of related measurements on the southern high latitude MR, we mirror the northern hemisphere $\mathrm{X}$-line to the south, indicated as the dashed line. The final result of the X-line configuration is quite similar to the prediction of Moore et al. [2002] given by maximizing the reconnecting component.

[18] It is worth mentioning that the equivalence between the FTE and the X-line orientation is based on 2D FTE generation theories/models. 3D flux ropes can easily have local orientations which differ greatly from the X-line orientation prior to FTE formation. This gives a potential source of error of the low-latitude X-line orientation. A similar problem exists in determining the high-latitude northern $\mathrm{X}$-line. In 3D situations, the $\mathrm{X}$-line may bend while elongating and therefore would be no longer perpendicular to the observed fast jets. It should also be noticed

Table 1. The Velocity, Axes and Maximum Core Field of the FTEs Observed by TC-1, All in GSM

\begin{tabular}{ccccc}
\hline Time (UT) & Velocity $(\mathrm{km} / \mathrm{s})$ & Axis & Axis “Clock Angle” & Core Field (nT) \\
\hline $04: 29$ & $-189,-81,-91$ & $0.421,0.883,-0.206$ & 103 & 67 \\
$04: 34$ & $-216,-141,-97$ & $0.631,0.605,-0.485$ & 128 & 48 \\
\hline
\end{tabular}


that the IMF had a notable $B_{X}$ component which would violate the south/north symmetry. Our result in the southern hemisphere should only be treated as a reference instead of representing the actual situation.

\section{Conclusion}

[19] In this study, a conjunction of Double Star TC-1 and Cluster on the dayside MP is analyzed. The five SCs traversed the MP within $20 \mathrm{~min}$ and encountered a series of FTEs and fast flows. Detailed studies show results as follows:

[20] 1. FTEs were observed by TC-1 at the southern hemisphere, not far away from the equator plane. The presence of a strong core field indicated that these FTEs were very likely related to component MR happening near the subsolar region.

[21] 2. A fast flow reversal and corresponding $B_{N}$ bipolar signature were detected by Cluster. Detailed analysis showed that Cluster passed nearby an anti-parallel MR region.

[22] 3. Due to the long duration of stable IMF condition, these two MR events can be considered as simultaneously happening but at different locations, and therefore a direct evidence of simultaneous occurrence of component and anti-parallel reconnections, agreeing well with previous theoretical and simulation works.

[23] Acknowledgments. We thank the teams of Cluster FGM and CIS and Double Star FGM and HIA for making the data available, and SPDF and NSSDC for providing the OMNI data. This work is supported by the NSFC grants 40731056, 40974095 and the Chinese Major Research Project (grant2011CB811404). We thank ISSI Team members and M. G. Kivelson and H. Zhang for useful discussions.

[24] The Editor thanks two anonymous reviewers for their assistance in evaluating this paper.

\section{References}

Berchem, J., et al. (2008), Reconnection at the dayside magnetopause: Comparisons of global MHD simulation results with Cluster and Double Star observations, J. Geophys. Res., 113, A07S12, doi:10.1029/ 2007JA012743.

Chandler, M. O., S. A. Fuselier, M. Lockwood, and T. E. Moore (1999), Evidence of component merging equatorward of the cusp, J. Geophys. Res., 104, 22,623-22,633, doi:10.1029/1999JA900175.

Cowley, S. W. H. (1973), A qualitative study of the reconnection between the Earth's magnetic field and an interplanetary field of arbitrary orientation, Radio Sci., 8, 903-913, doi:10.1029/RS008i011p00903.

Crooker, N. U. (1979), Dayside merging and cusp geometry, J. Geophys. Res., 84, 951-959, doi:10.1029/JA084iA03p00951.

Dorelli, J. C., A. Bhattacharjee, and J. Raeder (2007), Separator reconnection at Earth's dayside magnetopause under generic northward interplanetary magnetic field conditions, J. Geophys. Res., 112, A02202, doi:10.1029/ 2006JA011877.
Dungey, J. W. (1961), Interplanetary magnetic field and the auroral zones, Phys. Rev. Lett., 6, 47-48, doi:10.1103/PhysRevLett.6.47.

Dunlop, M. W., et al. (2005), Coordinated Cluster/Double Star observations of dayside reconnection signatures, Ann. Geophys., 23, 2867-2875.

Dunlop, M. W., Q. Zhang, C. Xiao, J. He, Z. Pu, R. C. Fear, C. Shen, and C. P. Escoubet (2009), Reconnection at high latitudes: Antiparallel merging, Phys. Rev. Lett., 102(7), 075005, doi:10.1103/PhysRevLett. 102.075005.

Fuselier, S. A., K. J. Trattner, and S. M. Petrinec (2000), Cusp observations of high- and low-latitude reconnection for northward interplanetary magnetic field, J. Geophys. Res., 105, 253-266, doi:10.1029/1999JA900422.

Gosling, J. T., M. F. Thomsen, S. J. Bame, R. C. Elphic, and C. T. Russell (1990), Plasma flow reversals at the dayside magnetopause and the origin of asymmetric polar cap convection, J. Geophys. Res., 95, 8073-8084, doi:10.1029/JA095iA06p08073.

Khrabrov, B. V., and B. U. O. Sonnerup (1998), Dehoffmann-teller analysis, in Analysis Methods for Multi-spacecraft Data, edited by G. Paschmann and P. Daly, ISSI Sci. Rep., SR-001, 221-248.

Lee, L. C., and Z. F. Fu (1985), A theory of magnetic flux transfer at the Earth's magnetopause, Geophys. Res. Lett., 12, 105-108, doi:10.1029/ GL012i002p00105.

Moore, T. E., M.-C. Fok, and M. O. Chandler (2002), The dayside reconnection X line, J. Geophys. Res., 107(A10), 1332, doi:10.1029/ 2002JA009381.

Onsager, T. G., J. D. Scudder, M. Lockwood, and C. T. Russell (2001), Reconnection at the high-latitude magnetopause during northward interplanetary magnetic field conditions, J. Geophys. Res., 106, 25,467-25,488, doi:10.1029/2000JA000444.

Paschmann, G. (2008), Recent in-situ observations of magnetic reconnection in near-Earth space, Geophys. Res. Lett., 35, L19109, doi:10.1029/ 2008 GL035297.

$\mathrm{Pu}, \mathrm{Z}$. Y., et al. (2005), Double Star TC-1 observations of component reconnection at the dayside magnetopause: A preliminary study, Ann Geophys., 23, 2889-2895.

$\mathrm{Pu}, \mathrm{Z}$. Y., et al. (2007), Global view of dayside magnetic reconnection with the dusk-dawn IMF orientation: A statistical study for Double Star and Cluster data, Geophys. Res. Lett., 34, L20101, doi:10.1029/ 2007GL030336.

Scholer, M. (1988), Magnetic flux transfer at the magnetopause based on single X line bursty reconnection, Geophys. Res. Lett., 15, 291-294, doi:10.1029/GL015i004p00291.

Sonnerup, B. U. O., and M. Scheible (1998), Minimum and maximum variance analysis, in Analysis Methods for Multi-spacecraft Data, edited by G. Paschmann and P. Daly, ISSI Sci. Rep., SR-001, 185-220.

Trattner, K. J., J. S. Mulcock, S. M. Petrinec, and S. A. Fuselier (2007), Location of the reconnection line at the magnetopause during southward IMF conditions, Geophys. Res. Lett., 34, L03108, doi:10.1029/ 2006 GL028397.

Wang, J., et al. (2008), Coordinated Cluster/Double Star observations of dayside flux transfer events on 6 April 2004, Sci. China Ser. E, 51, 1611-1619, doi:10.1007/s11431-008-0265-8.

Y. V. Bogdanova, Mullard Space Science Laboratory, University College London, Dorking RH5 6NT, UK.

M. W. Dunlop, Space Science and Technology Department, Rutherford Appleton Laboratory, Chilton OX11 0QX, UK.

S. Y. Fu, Z. Y. Pu, J. Wang, Y. Wei, L. Xie, and Q. G. Zong, School of Earth and Space Sciences, Peking University, 5 Yiheyuan St., Beijing 100871, China.(zypu@pku.edu.cn)

X. G. Wang and C. J. Xiao, School of Physics, Peking University, Beijing 100871, China. 